\title{
Fatores associados à autoavaliação negativa da saúde em idosos cadastrados nas Unidades Básicas de Saúde
}

\author{
Factors associated with negative self-assessment of health in elderly \\ enrolled in Primary Care Units
}

Eliziária Cardoso dos Santos¹, Bárbara de Menezes Couto², Alessandra de Carvalho Bastone²

${ }^{1}$ Faculdade de Medicina, Universidade Federal dos Vales do Jequitinhonha e Mucuri (UFVJM) - Diamantina (MG), Brasil.

${ }^{2}$ Faculdade de Ciências Básicas e da Saúde, Universidade Federal dos Vales do Jequitinhonha e Mucuri (UFVJM) - Diamantina (MG), Brasil.

DOI: https://dx.doi.org/10.7322/abcshs.v43i1.999

\section{RESUMO}

Introdução: A autoavaliação do estado de saúde pode ser considerada um preditor significante e independente de funcionalidade e morbimortalidade em idosos. Objetivo: Verificar a associação de indicadores sociodemográficos, de saúde e funcionalidade com a autoavaliação negativa da saúde (AANS) em idosos. Métodos: Estudo transversal, com uma amostra estratificada de idosos comunitários do município de Diamantina (MG), Brasil. Para avaliar a AANS e as variáveis sociodemográficas, de saúde e funcionalidade foi utilizado - Questionário BOAS - Brazil Old Age Schedule. Os testes Timed up \& go e Functional reach foram aplicados para avaliar funcionalidade e risco de quedas, respectivamente. Para as análises univariadas utilizou-se o teste Qui-Quadrado de Pearson e o teste Qui-Quadrado de tendência linear $(p \leq 0,20)$. As análises múltiplas foram realizadas por meio de três modelos de regressão logística $(p \leq 0,05)$. Resultados: 401 idosos participaram deste estudo. As variáveis que apresentaram associação significativa com a AANS foram: idade de 80 anos e mais $(\mathrm{OR}=1,81 ; \quad I C 95 \%=1,06-3,08)$, recurso financeiro insuficiente $(\mathrm{OR}=2,43 ; \quad \mathrm{IC} 95 \%=1,55-3,78)$, histórico de quedas (OR=3,24; IC95\%=1,71-6,14), hipertensão (OR=3,27; IC95\%=1,37-7,81), doença neurológica $(\mathrm{OR}=2,36$; IC95\%=1,18-4,71), doença osteomioarticular $(\mathrm{OR}=2,18$; IC95\%=1,26-3,78), depressão (OR=3,87; IC95\%=1,88$7,75)$, tempo de doença $>12$ anos $(\mathrm{OR}=2,13$; IC95\%=1,12-4,03), uso de prótese dentária $(\mathrm{OR}=0,50 ; \mathrm{IC} 95 \%=0,28-0,89)$, tempo $\geq 10$ segundos para realização do Timed up \& go $(\mathrm{OR}=2,54$; IC95\%=1,623,10), incapacidade em 2 ou mais atividades de vida diária $(\mathrm{OR}=1,86$; IC95\%=1,17-2,95) e autorrelato de sentir menos energia $(\mathrm{OR}=2,60 ; \mathrm{IC} 95 \%=1,57-4,31)$. Conclusão: A AANS está associada a fatores sociodemográficos, de saúde e funcionalidade, indicando a necessidade de um cuidado integral à saúde do idoso.

Palavras-chave: autoavaliação; idoso fragilizado; doença crônica; idoso; monitoramento epidemiológico; assistência integral à saúde do idoso.

\begin{abstract}
Introduction: The self-assessment of health might be considered a significant and independent predictor of physical function, morbidity and mortality in older adults. Objective: To verify the association between sociodemographic, health and physical functional data with negative self-assessment of health in elderly. Methods: Cross-sectional study, with a stratified sampling of communitydwelling from Diamantina (MG), Brazil. To evaluate the AANS and sociodemographic, health and functional variables Questionnaire the Brazil Old Age Schedule (BOAS). The Timed up \& go test and the Functional Reach test were performed to assess physical function and risk of falls, respectively. Chi-square tests were used to analyze the univariate associations $(p \leq 0.20)$. Multiple logistic regression models were created to identify the adjusted associated factors $(p \leq 0.05)$. Results: 401 older adults participated in this study. The variables that showed a significant association with the negative selfassessment of health were: 80 years or more $(\mathrm{OR}=1.81$; IC95\% $=1.06$ 3.08), insufficient financial resource $(\mathrm{OR}=2.43$; IC95\%=1.55-3.78), history of falls $(\mathrm{OR}=3.24$; $\mathrm{IC} 95 \%=1.71-6.14)$, hypertension $(\mathrm{OR}=3.27$; IC95\%=1.37-7.81), neurological disease $(\mathrm{OR}=2.36$; IC95\%=1.184.71), osteomioarticular disease $(\mathrm{OR}=2.18 ; \quad \mathrm{IC} 95 \%=1.26-3.78)$, depression $(\mathrm{OR}=3.87 ; \mathrm{IC} 95 \%=1.88-7.75)$, time of disease $>12$ years $(\mathrm{OR}=2.13$; IC95\%=1.12-4.03), use of dental prosthesis $(\mathrm{OR}=0.50$; IC95\%=0.28-0.89), time $\geq 10$ seconds to perform the Timed up \& go $(\mathrm{OR}=2.54$; IC95\%=1.62-3.10), disability in two or more activities of daily living $(\mathrm{OR}=1.86$; IC95\%=1.17-2.95) and self-report of less energy (OR=2.60; IC95\%=1.57-4.31). Conclusion: The negative self-assessment of health was associated with sociodemographic, health and physical function factors, indicating the need of a comprehensive health care for this population.
\end{abstract}

Keywords: self-assessment; frail elderly; chronic disease; aged; epidemiological monitoring; comprehensive health care. 


\section{INTRODUÇÃO}

O crescimento da população idosa representa uma realidade demográfica significativa no Brasil e no mundo ${ }^{1}$. Estudos de base populacional têm apontado que a quantidade de idosos, em números relativos e absolutos, está crescendo mais rapidamente do que qualquer outro segmento etário ${ }^{1,2}$. Esse novo perfil demográfico, além de evidenciar marcada alteração na estrutura social do país, confronta com uma maior probabilidade de vulnerabilidades e desenvolvimento de incapacidades associadas ao envelhecimento, o que acentua um novo perfil de morbimortalidade com reflexo direto no aumento dos custos com assistência à saúde $\mathrm{e}^{3,4}$.

Diante desse cenário, a incorporação de ferramentas capazes de estimar e monitorar as condições de saúde entre os idosos tornam-se altamente relevantes ${ }^{5,6}$. De acordo com Pimenta et al. ${ }^{5}$, uma estratégia chave fundamenta-se no autorrelato do estado geral da saúde. Estudos têm apontado que a autoavaliação da saúde, além de contribuir para o levantamento das principais afecções que acometem os diferentes estratos populacionais, pode fundamentar o processo de avaliação e efetividade das políticas públicas em saúde, de acordo com as peculiaridades epidemiológicas apresentadas pelos indivíduos em diferentes regiões do país $^{6-9}$.

Baseado nesse fato, Pagotto et al. ${ }^{6}$ relataram que a autoavaliação do estado de saúde pode ser considerado um preditor significativo e independente para avaliar a funcionalidade e morbimortalidade entre idosos, além de ser um indicador confiável das condições de saúde, capaz de contemplar os domínios da saúde física, cognitiva e emocional dos indivíduos. Adicionalmente, estudos epidemiológicos têm apontado que a percepção que os indivíduos têm sobre sua própria condição de saúde pode apresentar relevante associação com seu bem estar geral, sendo útil para avaliar as necessidades de saúde e predizer sua sobrevida, uma vez que a maneira como o indivíduo lida com seu estado de saúde pode determinar seu comportamento, suas escolhas, seu bem estar e seu modo de viver ${ }^{5,10,11}$.

Apesar da relevância do uso dessa ferramenta como parâmetro de pesquisa na área da saúde, especialmente em idosos, ela ainda necessita ser explorada para analisar a saúde sob a ótica do idoso em regiões de alta vulnerabilidade social, como o Vale do Jequitinhonha, localizado no norte do Estado de Minas Gerais ${ }^{12}$.

Nesse sentido, o presente estudo objetivou verificar a associação entre indicadores sociodemográficos, de saúde e funcionalidade, com a variável dependente autoavaliação negativa da saúde (AANS), em idosos cadastrados nas Unidades Básicas de Saúde do município de Diamantina/ Vale do Jequitinhonha, MG.

\section{MÉTODOS}

Tratou-se de um estudo transversal, baseado em inquérito domiciliar, com amostra aleatória estratificada, representativa dos idosos ( $\geq 60$ anos) cadastrados em sete Unidades Básicas de
Saúde (UBS) do município de Diamantina, situado na mesorregião do Vale do Jequitinhonha/MG. Esta pesquisa seguiu os princípios éticos presentes na Declaração de Helsinque. O projeto foi aprovado pelo Comitê de Ética em Pesquisa com Seres Humanos da Universidade Federal de Diamantina, protocolo número 005/2008.

A amostra populacional foi calculada a partir de um número de 1975 idosos cadastrados nas sete UBS, em 2008, por meio do programa EPI INFO 7.1.5.2, utilizando como parâmetros: nível de confiança de $95 \%$, prevalência de autoavaliação negativa em saúde de $30 \%$ e erro amostral de 4,5 pontos percentuais. Adicionou-se $10 \%$ com o propósito de compensar possíveis perdas e $20 \%$ como controle de fatores de confusão totalizando 429 indivíduos.

O procedimento utilizado para a seleção amostral procedeu-se em duas etapas subsequentes:

1. Após a realização do cálculo amostral, descrito acima, o passo seguinte foi selecionar as ruas e casas a serem visitadas nos respectivos bairros. Cada bairro visitado foi dividido em quatro quadras, de acordo com a divisão já realizada pelas agentes de saúde para guiar suas visitas domiciliares. Para determinar o número de domicílios por quadra, o número de idosos/área de abrangência do UBS foi dividido por quatro, de modo que um número proporcional de idosos pudesse ser entrevistado em cada uma das quadras. Para saber qual seria o primeiro domicílio/quadra com idoso cadastrado na respectiva UBS a ser visitado realizou-se um sorteio. O mesmo procedimento foi realizado para os demais domicílios em cada quadra sucessivamente até atingir a totalidade do número amostral de idosos residentes na localidade específica. Os domicílios já sorteados foram excluídos dos sorteios subsequentes.

2. Nos domicílios em que o idoso estivesse ausente, no momento da visita, optou-se pelo agendamento de um horário específico e, se no dia agendado o idoso não fosse encontrado na residência, considerava-se o domicílio subsequente. Foi realizado um sorteio quando, na residência selecionada, havia mais de um idoso. Os idosos selecionados foram esclarecidos a respeito dos objetivos da pesquisa e aqueles que concordaram voluntariamente em participar, assinaram o termo de consentimento livre e esclarecido.

A coleta dos dados foi realizada no período de 2008 a 2009 e procedeu-se utilizando um instrumento estruturado na forma de questionário multidimensional, organizado em oito sessões e, desenvolvido especificamente para pesquisas com populações idosas (Questionário BOAS - Brazil Old Age Schedule) de Veras et al. ${ }^{13}$. Além disso, foram realizados os testes Timed up \& $g o^{14}$, para avaliar a funcionalidade dos indivíduos, e o Functional reach test ${ }^{15}$, para avaliar o risco de quedas dos participantes. Foi adotado como critério de inclusão idade igual ou maior do que 60 anos, viver em comunidade e ser capaz de deambular de forma independente, com ou sem auxílio para marcha. Foram excluídos idosos que apresentaram déficit cognitivo, avaliado por meio do Mini Exame do Estado Mental (MEEM) ${ }^{16}$. 
Para garantir a homogeneidade dos dados coletados, os pesquisadores e voluntários foram previamente treinados de acordo com as instruções padronizadas para aplicação do questionário multidimensional e dos testes funcionais.

Após a coleta dos dados determinou-se como variável dependente a autoavaliação negativa da saúde, obtida por meio da seguinte pergunta: "Em geral, o (a) Sr.(a) diria que sua saúde está?" As respostas "ótima e boa" foram agrupadas na categoria de autoavaliação positiva de saúde e as respostas "ruim e péssima" foram classificadas como autoavaliação negativa de saúde.

As variáveis independentes incluídas no estudo foram divididas em três níveis: sociodemográficas, de saúde e de funcionalidade. Estas variáveis, seus níveis, tipos e formas de categorização/pontos de corte estão descritas no Quadro 1.

Os dados descritivos da amostra foram apresentados por meio de frequência absoluta e frequência relativa. Para verificar possíveis associações entre a variável dependente, categorizada como autoavaliação negativa da saúde, e cada variável independente, utilizou-se o teste Qui-Quadrado de Pearson e o teste QuiQuadrado de tendência linear. As variáveis com $p \leq 0,20$ nestes testes foram selecionadas para inclusão nas análises múltiplas e divididas em três modelos: variáveis sociodemográficas, variáveis de saúde e variáveis de funcionalidade. As análises múltiplas foram realizadas por meio de regressão logística múltipla, sendo estimadas as razões de chances (odds ratio) e os respectivos intervalos de confiança de $95 \%$. O critério de permanência das variáveis nos modelos finais foi $p \leq 0,05$. Para as análises, utilizou-se o software SPSS v. 19.0.

\section{RESULTADOS}

A partir do número total de idosos elegíveis cadastrados nas sete UBS do município de Diamantina, MG $(\mathrm{n}=1975)$, um total de 429 indivíduos foram visitados em suas respectivas residências. Desses, 28 (6,5\%) idosos foram excluídos por apresentarem déficit cognitivo, totalizando uma amostra analítica de 401 indivíduos. Dessa amostra, o maior percentual de idosos era constituído por mulheres $(65,3 \%)$, encontravam-se na faixa etária categorizada no intervalo de 60 - 69 anos de idade (42,1\%), eram casados (43,6\%) e apresentavam de 1 a 4 anos de escolaridade (51,6\%) (Tabela 1).

Quadro 1: Descrição das variáveis dependentes do estudo

\begin{tabular}{|c|c|c|c|}
\hline Nível & Variável & Tipo & Descrição \\
\hline \multirow{7}{*}{ 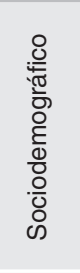 } & Sexo & Dicotômica & Feminino / Masculino \\
\hline & Idade & Coletada de forma contínua e categorizada & $60-69 ; 70-79 ; 80+$ \\
\hline & Estado conjugal & Categórica & Casado; Viúvo; Divorciado/separado; Solteiro \\
\hline & Escolaridade & Coletada de forma contínua e categorizada & Nenhuma; $1-4$ anos; 5-8 anos; 9 - 11 anos; > 11 anos \\
\hline & Mora só & Dicotômica & Sim / não \\
\hline & Recurso Financeiro & Dicotômica & Suficiente / Insuficiente \\
\hline & Residência & Coletada de forma categórica e dicotomizada & Ótima, boa / Ruim, péssima \\
\hline \multirow{22}{*}{ 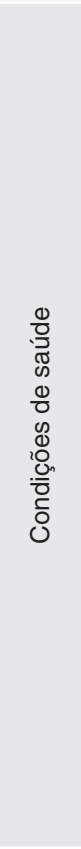 } & Incontinência urinária & Dicotômica & Sim / Não \\
\hline & Déficit visual & Dicotômica & Sim / Não \\
\hline & Déficit auditivo & Dicotômica & Sim / Não \\
\hline & Histórico de quedas & Dicotômica & Sim / Não \\
\hline & Hipertensão & Dicotômica & Sim / Não \\
\hline & Diabetes & Dicotômica & Sim / Não \\
\hline & Dislipidemia & Dicotômica & Sim / Não \\
\hline & Doença respiratória & Dicotômica & Sim / Não \\
\hline & Doença renal & Dicotômica & Sim / Não \\
\hline & Doença cardíaca & Dicotômica & Sim / Não \\
\hline & Doença gatrointestinal & Dicotômica & Sim / Não \\
\hline & Doença neurológica & Dicotômica & Sim / Não \\
\hline & Doença osteomioarticular & Dicotômica & Sim / Não \\
\hline & Depressão & Dicotômica & Sim / Não \\
\hline & Insônia & Dicotômica & Sim / Não \\
\hline & Comorbidade & Dicotômica & Sim / Não \\
\hline & Tempo de doença & Coletada de forma contínua e categorizada & Nenhum ; $\leq 12$ meses; > 12 meses \\
\hline & Saúde bucal & Coletada de forma categorizada e dicotomizada & Ótima, boa / Regular, ruim \\
\hline & Possui prótese dentária & Dicotômica & Sim / Não \\
\hline & Sentimento de solidão & Dicotômica & Sim / Não \\
\hline & Preocupação & Dicotômica & Sim / Não \\
\hline & Medicamento & Coletada de forma contínua e categorizada & Nenhum; $1-2 ; \geq 3$ \\
\hline \multirow{5}{*}{$\begin{array}{l}\frac{0}{0} \\
\frac{\pi}{0} \\
\frac{0}{\widetilde{0}} \\
\stackrel{0}{0} \\
\stackrel{0}{0} \\
\stackrel{5}{J}\end{array}$} & Timed up \& go & Coletada de forma contínua e dicotomizada & $\leq 10$ segundos / > 10 segundos \\
\hline & Incapacidade nas AVD & Coletada de forma contínua e dicotomizada & $0-1 />1$ \\
\hline & Ajuda nas AVD & Dicotômica & Sim / Não \\
\hline & Functional reach test & Coletada de forma contínua e dicotomizada & $\geq 15$ centímetros / < 15 centímetros \\
\hline & Autorrelato de menos energia & Dicotômica & Sim / Não \\
\hline
\end{tabular}


Tabela 1: Distribuição da amostra e prevalência da autoavaliação negativa da saúde de acordo com cada variável independente, por nível de classificação: variáveis sociodemográficas, de saúde e de funcionalidade em idosos cadastrados nas UBS da área de abrangência do estudo. Diamantina, MG, Brasil, 2009. $(n=401)$

\begin{tabular}{|c|c|c|c|c|}
\hline Nível & Variável & n (\%) & $\begin{array}{c}\text { Prevalência } \\
\text { AANS }\end{array}$ & Valor $p$ \\
\hline \multirow{27}{*}{ 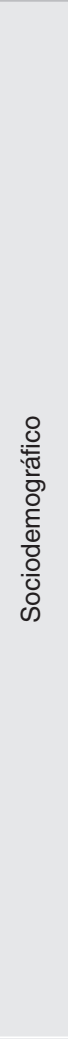 } & \multicolumn{4}{|l|}{$\mathrm{Sexo}^{\dagger}$} \\
\hline & Feminino & $262(65,3)$ & 37,4 & 0,148 \\
\hline & Masculino & $139(34,7)$ & 30,2 & \\
\hline & \multicolumn{4}{|l|}{ Idade ${ }^{t \dagger}$} \\
\hline & $60-69$ & $169(42,1)$ & 27,2 & 0,006 \\
\hline & $70-79$ & $150(37,4)$ & 36,7 & \\
\hline & $80+$ & $82(20,5)$ & 47,6 & \\
\hline & \multicolumn{4}{|l|}{ Estado conjugal $^{\dagger+}$} \\
\hline & Casado & $175(43,6)$ & 33,7 & 0,962 \\
\hline & Viúvo & $174(43,4)$ & 35,6 & \\
\hline & Divorciado/separado & $20(5,0)$ & 35,0 & \\
\hline & Solteiro & $32(8,0)$ & 37,5 & \\
\hline & \multicolumn{4}{|l|}{ Escolaridade $^{\dagger \dagger}$ (anos) } \\
\hline & Nenhuma & $120(29,9)$ & 45,8 & 0,012 \\
\hline & $1-4$ & $207(51,6)$ & 31,4 & \\
\hline & $5-8$ & $41(10,2)$ & 24,4 & \\
\hline & $9-11$ & $19(4,7)$ & 42,1 & \\
\hline & $>11$ & $14(3,6)$ & 14,3 & \\
\hline & \multicolumn{4}{|l|}{ Mora só ${ }^{\dagger}$} \\
\hline & Sim & $51(12,7)$ & 3,9 & 0,493 \\
\hline & Não & $350(87,3)$ & 34,3 & \\
\hline & \multicolumn{4}{|l|}{ Recurso financeiro $^{\dagger}$} \\
\hline & Suficiente & $229(57,1)$ & 27,5 & 0,001 \\
\hline & Insuficiente & $168(41,9)$ & 43,5 & \\
\hline & \multicolumn{4}{|l|}{ Residência $^{\dagger}$} \\
\hline & Ótima, boa & $302(75,3)$ & 34,8 & 0,916 \\
\hline & Ruim, péssima & $99(24,7)$ & 35,4 & \\
\hline \multirow{30}{*}{ 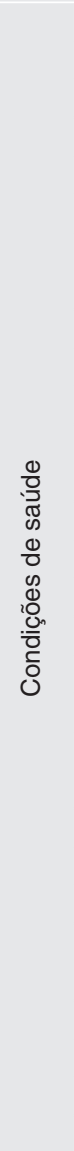 } & \multicolumn{4}{|l|}{ Incontinência urinária ${ }^{\dagger}$} \\
\hline & Sim & $124(30,9)$ & 42,7 & 0,029 \\
\hline & Não & $277(69,1)$ & 31,4 & \\
\hline & \multicolumn{4}{|l|}{ Déficit visual ${ }^{\dagger}$} \\
\hline & Sim & $82(20,5)$ & 45,1 & 0,032 \\
\hline & Não & $319(79,5)$ & 32,3 & \\
\hline & \multicolumn{4}{|l|}{ Déficit auditivo $^{\dagger}$} \\
\hline & Sim & $94(23,4)$ & 46,8 & 0,006 \\
\hline & Não & $307(76,6)$ & 31,3 & \\
\hline & \multicolumn{4}{|l|}{ Histórico de quedas ${ }^{\dagger}$} \\
\hline & Sim & $66(16,5)$ & 60,6 & 0,001 \\
\hline & Não & $334(83,3)$ & 29,6 & \\
\hline & \multicolumn{4}{|l|}{ Hipertensão $^{\dagger}$} \\
\hline & Sim & $329(82,1)$ & 38,6 & 0,001 \\
\hline & Não & $72(17,9)$ & 18,1 & \\
\hline & \multicolumn{4}{|l|}{ Diabetes $^{\dagger}$} \\
\hline & Sim & $119(29,7)$ & 36,1 & 0,739 \\
\hline & Não & $282(70,3)$ & 34,4 & \\
\hline & \multicolumn{4}{|l|}{ Dislipidemia $^{\dagger}$} \\
\hline & Sim & $76(19,0)$ & 38,2 & 0,512 \\
\hline & Não & $325(81,0)$ & 34,2 & \\
\hline & \multicolumn{4}{|l|}{ Doença respiratória $^{\dagger}$} \\
\hline & Sim & $18(4,5)$ & 22,2 & 0,384 \\
\hline & Não & $383(95,5)$ & 35,5 & \\
\hline & \multicolumn{4}{|l|}{ Doença renal ${ }^{\dagger}$} \\
\hline & Sim & $10(2,5)$ & 40,0 & 0,735 \\
\hline & Não & $391(97,5)$ & 34,8 & \\
\hline & Doença cardíaca $^{\dagger}$ & & & \\
\hline & Sim & $62(15,5)$ & 40,3 & 0,335 \\
\hline & Não & $339(84,5)$ & 33,9 & \\
\hline
\end{tabular}

\begin{tabular}{|c|c|c|c|c|}
\hline Nível & Variável & n (\%) & $\begin{array}{c}\text { Prevalência } \\
\text { AANS }\end{array}$ & Valor $p$ \\
\hline \multirow{38}{*}{ 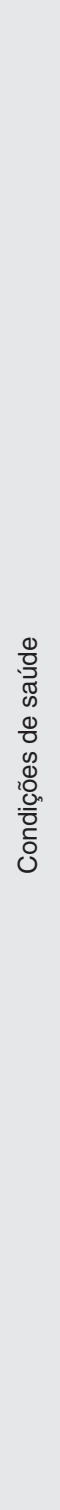 } & \multicolumn{4}{|l|}{ Doença gastrointestinal $^{+}$} \\
\hline & Sim & $19(4,7)$ & 36,8 & 0,857 \\
\hline & Não & $382(95,3)$ & 34,8 & \\
\hline & \multicolumn{4}{|l|}{ Doença neurológica $^{\dagger}$} \\
\hline & Sim & $60(15,0)$ & 45,0 & 0,080 \\
\hline & Não & $341(85,0)$ & 33,1 & \\
\hline & \multicolumn{4}{|l|}{ Doença osteomioarticular ${ }^{\dagger}$} \\
\hline & Sim & $154(38,4)$ & 42,9 & 0,009 \\
\hline & Não & $247(61,6)$ & 30,0 & \\
\hline & \multicolumn{4}{|l|}{ Depressão $^{\dagger}$} \\
\hline & Sim & $54(13,5)$ & 64,8 & 0,001 \\
\hline & Não & $347(86,5)$ & 30,3 & \\
\hline & \multicolumn{4}{|l|}{ Insônia $^{\dagger}$} \\
\hline & Sim & $156(38,9)$ & 45,5 & 0,001 \\
\hline & Não & $245(61,1)$ & 28,2 & \\
\hline & \multicolumn{4}{|l|}{ Comorbidade $^{\dagger}$} \\
\hline & Sim & $282(70,3)$ & 40,1 & 0,001 \\
\hline & Não & $119(29,7)$ & 22,7 & \\
\hline & \multicolumn{4}{|c|}{ Tempo de doença ${ }^{\dagger \dagger}$ (meses) } \\
\hline & Nenhum & $84(21,0)$ & 23,8 & 0,001 \\
\hline & $\leq 12$ & $225(56,1)$ & 32,4 & \\
\hline & $>12$ & $92(22,9)$ & 51,1 & \\
\hline & \multicolumn{4}{|l|}{ Saúde Bucal ${ }^{\dagger}$} \\
\hline & Ótima, boa & $44(11,0)$ & 38,6 & 0,832 \\
\hline & Regular, ruim & $354(88,3)$ & 34,5 & \\
\hline & \multicolumn{4}{|l|}{ Possui prótese dentária $^{\dagger}$} \\
\hline & Sim & $313(78,1)$ & 31,0 & 0,005 \\
\hline & Não & $86(21,5)$ & 47,7 & \\
\hline & \multicolumn{4}{|l|}{ Sentimento de solidão ${ }^{\dagger}$} \\
\hline & Sim & $126(31,4)$ & 44,4 & 0,006 \\
\hline & Não & $274(68,3)$ & 30,3 & \\
\hline & \multicolumn{4}{|l|}{ Preocupação $^{\dagger}$} \\
\hline & Sim & $284(70,8)$ & 36,6 & 0,260 \\
\hline & Não & $114(28,4)$ & 30,7 & \\
\hline & \multicolumn{4}{|l|}{ Medicamento ${ }^{\dagger \dagger}$} \\
\hline & Nenhum & $72(18,0)$ & 22,2 & 0,007 \\
\hline & $1-2$ & $271(67,6)$ & 35,4 & \\
\hline & $\geq 3$ & $58(14,5)$ & 48,3 & \\
\hline \multirow{15}{*}{$\begin{array}{l}\frac{0}{0} \\
\frac{\pi}{0} \\
\frac{0}{\overline{0}} \\
\frac{0}{0} \\
\frac{0}{0} \\
\frac{5}{5} \\
4\end{array}$} & \multicolumn{4}{|c|}{ Timed up \& $\mathrm{go}^{\dagger}$ (segundos) } \\
\hline & $\leq 10$ & $218(54,3)$ & 23,9 & 0,001 \\
\hline & $>10$ & $182(45,4)$ & 47,8 & \\
\hline & \multicolumn{4}{|l|}{ Incapacidade nas AVD ${ }^{\dagger}$} \\
\hline & $0-1$ & $248(61,8)$ & 28,2 & 0,001 \\
\hline & $>1$ & $153(38,2)$ & 47,8 & \\
\hline & \multicolumn{4}{|l|}{ Ajuda nas $\mathrm{AVD}^{\dagger}$} \\
\hline & Sim & $291(72,6)$ & 37,5 & 0,079 \\
\hline & Não & $110(27,4)$ & 28,2 & \\
\hline & \multicolumn{4}{|c|}{ Functional reach test ${ }^{\dagger}$ (centímetros) } \\
\hline & $\geq 15$ & $348(86,8)$ & 33,3 & 0,144 \\
\hline & $<15$ & $50(12,5)$ & 44,0 & \\
\hline & \multicolumn{4}{|c|}{ Autorrelato de menos energia $^{\dagger}$} \\
\hline & Sim & $260(64,8)$ & 42,3 & 0,001 \\
\hline & Não & $138(34,4)$ & 20,3 & \\
\hline
\end{tabular}

AANS: Autoavaliação negativa da saúde; AVD: atividades de vida diária; ‘Teste qui-quadrado de Pearson; †TTeste qui-quadrado de tendência linear. 
$\mathrm{Na}$ análise univariada, as variáveis que apresentaram associação significativa $(p \leq 0,20)$ com a autoavaliação negativa da saúde foram: sexo, idade, escolaridade, recurso financeiro, incontinência urinária, déficit visual, déficit auditivo, histórico de quedas, hipertensão, doença neurológica, doença osteomioarticular, depressão, insônia, comorbidade, tempo de doença, uso de prótese dentária total ou parcial, sentimento de solidão, medicamento, Timed up \& go, incapacidade nas atividades de vida diária, ajuda para realizar as atividades de vida diária, Functional reach test e autorrelato de menos energia (Tabela 1).

Após ajuste para correção de fatores de confusão, as análises de regressão logística múltipla mostraram que: em relação às variáveis sociodemográficas, apenas idade de 80 anos e mais (OR $=1,81 ; \mathrm{IC} 95 \%=1,06-3,08)$ e recurso financeiro insuficiente $(\mathrm{OR}$ $=2.43$; IC $95 \%=1,55-3,78$ ) permaneceram significativamente associadas à autoavaliação negativa da saúde (Tabela 2). No modelo que englobou as variáveis de saúde, histórico de quedas (OR $=3,24$; IC95\% = 1,71-6,14), hipertensão $(\mathrm{OR}=3,27$; IC95\% = $1,37-7,81)$, doença neurológica $(\mathrm{OR}=2,36$; IC95\% = 1,18-4,71), doença osteomioarticular ( $\mathrm{OR}=2,18$; IC95\% = 1,26-3,78), depressão ( $\mathrm{OR}=3,87$; IC95\% = 1,88-7,75), tempo de doença maior do que doze meses $(\mathrm{OR}=2,13$; IC95\% $=1,12-4,03)$ e uso de prótese dentária total ou parcial $(\mathrm{OR}=0,50$; IC95\% $=0,28-0,89)$ mantiveram associação significativa com autoavaliação negativa da saúde (Tabela 3). Em relação às variáveis de funcionalidade, tempo maior que dez segundos para realização do teste Timed up \& go $(\mathrm{OR}=2,54$; IC95\% = 1,62-3,10), incapacidade para realizar 2 ou mais atividades de vida diária $(\mathrm{OR}=1,86$; IC95\% $=1,17$ $2,95)$ e o autorrelato de sentir menos energia durante o dia $(\mathrm{OR}=$ 2,60; IC95\% = 1,57-4,31) mantiveram associação estatisticamente

Tabela 2: Análise multivariada das variáveis sociodemográficos associadas à autoavaliação negativa da saúde em idosos cadastrados nas UBS da área de abrangência do estudo. Diamantina, MG, Brasil, 2009. $(n=401)$

\begin{tabular}{|c|c|c|c|}
\hline Variável & OR & IC $95 \%$ & Valor $p$ \\
\hline \multicolumn{4}{|l|}{ Sexo } \\
\hline Masculino & 1 & & \\
\hline Feminino & 1,312 & $0,822-2,093$ & 0,255 \\
\hline \multicolumn{4}{|l|}{ Idade } \\
\hline $60-69$ & 1 & & \\
\hline $70-79$ & 1,602 & $0,976-2,603$ & 0,062 \\
\hline $80+$ & 1,805 & $1,060-3,075$ & 0,030 \\
\hline \multicolumn{4}{|c|}{ Escolaridade (anos) } \\
\hline Nenhuma & 1 & & \\
\hline $1-4$ & 0,692 & $0,424-1,129$ & 0,140 \\
\hline $5-8$ & 0,534 & $0,240-1,188$ & 0,124 \\
\hline $9-11$ & 2,084 & $0,756-5,745$ & 0,156 \\
\hline$>11$ & 0,397 & $0,083-1,889$ & 0,245 \\
\hline \multicolumn{4}{|c|}{ Recurso financeiro } \\
\hline Suficiente & 1 & & \\
\hline Insuficiente & 2,425 & $1,552-3,788$ & 0,001 \\
\hline
\end{tabular}

OR: Odds Ratio; IC: Intervalo de Confiança de 95\%. significativa com a variável dependente, autoavaliação negativa do estado de saúde (Tabela 4). Todas as condições citadas acima estavam associadas a uma maior chance do idoso avaliar sua saúde como ruim ou péssima, com exceção do uso de prótese dentária total ou parcial, podendo este ser considerado um fator de proteção em relação à autoavaliação negativa da saúde.

Tabela 3: Análise multivariada das variáveis de saúde associadas à autoavaliação negativa da saúde em idosos cadastrados nas UBS da área de abrangência do estudo. Diamantina, MG, Brasil, 2009. $(n=401)$

\begin{tabular}{|c|c|c|c|}
\hline Variável independente & OR & IC $95 \%$ & Valor $p$ \\
\hline \multicolumn{4}{|l|}{ Incontinência urinária } \\
\hline Sim & 1,022 & $0,608-1,171$ & 0,934 \\
\hline Não & 1 & & \\
\hline \multicolumn{4}{|l|}{ Déficit visual } \\
\hline Sim & 1,556 & $0,877-2,758$ & 0,130 \\
\hline Não & 1 & & \\
\hline \multicolumn{4}{|l|}{ Déficit auditivo } \\
\hline Sim & 1,542 & $0,884-2,687$ & 0,127 \\
\hline Não & 1 & & \\
\hline \multicolumn{4}{|l|}{ Histórico de quedas } \\
\hline Sim & 3,240 & $1,710-6,136$ & 0,001 \\
\hline Não & 1 & & \\
\hline \multicolumn{4}{|l|}{ Hipertensão } \\
\hline $\operatorname{Sim}$ & 3,269 & $1,367-7,814$ & 0,008 \\
\hline Não & 1 & & \\
\hline \multicolumn{4}{|l|}{ Doença neurológica } \\
\hline Sim & 2,362 & $1,183-4,713$ & 0,015 \\
\hline Não & 1 & & \\
\hline \multicolumn{4}{|l|}{ Doença osteomioarticular } \\
\hline Sim & 2,178 & $1,255-3,779$ & 0,006 \\
\hline Não & 1 & & \\
\hline \multicolumn{4}{|l|}{ Comorbidade } \\
\hline Sim & 1,126 & $0,556-2,278$ & 0,742 \\
\hline Não & 1 & & \\
\hline \multicolumn{4}{|l|}{ Tempo de doença (meses) } \\
\hline Nenhum & 1 & & \\
\hline$\leq 12$ & 0,876 & $0,393-1,953$ & 0,746 \\
\hline$>12$ & 2,128 & $1,124-4,027$ & 0,020 \\
\hline \multicolumn{4}{|l|}{ Possui prótese dentária } \\
\hline Sim & 0,503 & $0,283-0,893$ & 0,019 \\
\hline Não & 1 & & \\
\hline \multicolumn{4}{|l|}{ Depressão } \\
\hline Sim & 3,871 & $1,884-7,954$ & 0,001 \\
\hline Não & 1 & & \\
\hline \multicolumn{4}{|l|}{ Insônia } \\
\hline Sim & 1,463 & $0,890-2,406$ & 0,133 \\
\hline Não & 1 & & \\
\hline \multicolumn{4}{|l|}{ Sentimento de solidão } \\
\hline Sim & 1,288 & $0,766-2,164$ & 0,340 \\
\hline Não & 1 & & \\
\hline \multicolumn{4}{|l|}{ Medicamento } \\
\hline Nenhum & 1 & & \\
\hline $1-2$ & 0,676 & $0,279-1,639$ & 0,386 \\
\hline$\geq 3$ & 0,740 & $0,338-1,622$ & 0,452 \\
\hline
\end{tabular}

OR: Odds Ratio; IC: Intervalo de Confiança de 95\%. 


\section{DISCUSSÃO}

O presente estudo analisou os fatores associados à autoavaliação negativa da saúde, em idosos comunitários cadastrados nas UBS do município de Diamantina, MG. De modo geral, nossos resultados identificaram associação entre a autoavaliação negativa da saúde com indicadores sociodemográficos, condições de saúde e diminuição da funcionalidade na população estudada.

A amostra apresentou uma maior proporção de mulheres e, de acordo com estudos prévios, há maior prevalência de mulheres nas faixas etárias acima de 60 anos ${ }^{17-19}$. De acordo com Almeida et al. ${ }^{20}$, os homens apresentam maior exposição a fatores de risco para mortalidade, pior atitude em relação ao processo saúde/ doença e inserção diferenciada no mercado de trabalho, determinando uma menor expectativa de vida quando comparado às mulheres em idade avançada.

O baixo nível de escolaridade, também esteve presente em nossa população. Geib ${ }^{19}$ e Soares \& Istoe ${ }^{21}$ ao estudar o processo de alfabetização nesse segmento etário, destacaram, que o mesmo reflete as políticas educacionais brasileiras remotas, na qual o ensino fundamental repercutia o processo de exclusão educacional, o que justifica o índice expressivo de analfabetismo em idosos. De acordo com o PNAD 22 , em 2015, o índice de analfabetismo no segmento etário de 60 anos ou mais, no Brasil, era de aproximadamente $23,1 \%$. Vale destacar que o Vale do Jequitinhonha é a região com maior índice de analfabetismo e analfabetismo funcional do Estado de Minas Gerais.

Relacionado aos indicadores já ressaltados, a idade avançada representa um fator relevante, que pode associar-se a agravos no perfil de saúde doença do idoso e que, quando agregada à ideia de baixa renda, advindas de aposentadoria ou da sua inexistência, influencia negativamente na maneira como esses indivíduos avaliam sua própria condição de saúde ${ }^{23}$, fato também relevante em

Tabela 4: Análise multivariada das variáveis de funcionalidade associadas à autoavaliação negativa da saúde em idosos cadastrados nas UBS da área de abrangência do estudo. Diamantina, MG, Brasil, 2009. $(n=401)$

\begin{tabular}{|c|c|c|c|}
\hline Variável independente & OR & IC $95 \%$ & Valor $p$ \\
\hline \multicolumn{4}{|c|}{ Timed up and GO (segundos) } \\
\hline$\leq 10$ & 1 & & \\
\hline$>10$ & 2,541 & $1,617-3,995$ & 0,001 \\
\hline \multicolumn{4}{|l|}{ Incapacidade nas AVDs } \\
\hline $0-1$ & 1 & & \\
\hline$>1$ & 1,859 & $1,170-2,954$ & 0,009 \\
\hline \multicolumn{4}{|l|}{ Ajuda nas AVDs } \\
\hline Sim & 1 & & \\
\hline Não & 1,115 & $0,661-1,882$ & 0,683 \\
\hline \multicolumn{4}{|c|}{ Functional reach test (centímetros) } \\
\hline$\geq 15$ & 1 & & \\
\hline$<15$ & 1,056 & $0,541-2,062$ & 0,873 \\
\hline \multicolumn{4}{|c|}{ Autorrelato de menos energia } \\
\hline Sim & 2,597 & $1,566-4,305$ & 0,001 \\
\hline Não & 1 & & \\
\hline
\end{tabular}

nossa investigação. Em um estudo realizado por Alves et al. ${ }^{24}$, a condição socioeconômica dos idosos, reflexo do grau de educação e a renda global, apresenta, forte relação com aspectos relevantes de sua saúde geral. Wickrama et al. ${ }^{25}$, em estudo prospectivo, delineado para avaliar a heterogeneidade da trajetória de saúde multidimensional de 1.945 idosos, ressaltaram que a condição socioeconômica dos idosos impactam de forma negativa suas relações sociais, bem como os hábitos de vida e o acesso aos serviços de saúde pública, fatores que em conjunto contribuem para que os mesmos avaliem de forma negativa a própria saúde.

O impacto da alta prevalência de doenças crônicas, associado ao tempo de doença, à redução da capacidade funcional e a um estado depressivo, tem sido sistematicamente relatado na literatura como fator capaz de influenciar negativamente a autoavaliação do estado de saúde do idoso ${ }^{23,24,26-28}$. Belém et al. ${ }^{29}$, em um estudo transversal de base domiciliar, delineado para avaliar fatores associados a autoavaliação negativa da saúde em indivíduos cadastrados nas Estratégias de Saúde da Família do Estado da Paraíba, encontraram associação positiva entre doenças crônicas, sentimento depressivo e autoavaliação negativa do estado de saúde em idosos. Na presente investigação, foi observada associação entre autoavaliação negativa da saúde e histórico de quedas, hipertensão, doença neurológica, doença osteomioarticular, depressão, tempo de doença maior do que doze meses e pior funcionalidade, caracterizada por um maior tempo para realização do teste Timed up \& go e por maior incapacidade para realizar atividades de vida diária.

Segundo Borim et al. ${ }^{28}$, a prevalência de doenças crônicas em idosos tende a um aumento considerável com o avançar da idade atingindo, aproximadamente, 70\% dos indivíduos acima de 70 anos de idade, sendo que a presença de mais de uma morbidade impacta negativamente a qualidade de vida e a autoavaliação da saúde desses indivíduos ${ }^{28,30,31}$. Em nosso estudo, apesar da variável comorbidade apresentar uma associação significativa com autoavaliação negativa da saúde na análise univariada, a significância da associação não se manteve no modelo multivariado.

Dentre as doenças estudadas, a depressão foi a que apresentou maior razão de chances para ocorrência de uma autoavaliação negativa da saúde e tem sido reportada como um importante determinante do declínio na autoavaliação da saúde ${ }^{32,33}$. Isso se justifica, uma vez que os idosos que apresentam associação de doenças crônicas com autoavaliação negativa de saúde tendem a maior prevalência de depressão, mesmo após ajuste pelas variáveis demográficas, socioeconômicas e de uso de serviços de saú$\mathrm{de}^{24}$. Além disso, conforme relatado por Alves \& Rodrigues ${ }^{24}$, a presença de doenças crônicas entre os idosos pode apresentar associação direta com um estado depressivo desses indivíduos fato que impacta na autoavaliação negativa da saúde entre os mesmos. Segundo Keyes e Westerhof ${ }^{34}$, apesar da idade avançada, o simples fato dos idosos relatarem que se sentem mais jovens, faz com que os mesmos tenham uma percepção otimista sobre sua própria saúde tanto física quanto mental e consequentemente diminui o risco da instalação precoce de incapacidades e de um estado 
depressivo, fatores que poderiam contribuir, consideravelmente, para uma boa percepção do próprio estado de saúde, o que não foi uma realidade na presente investigação.

Ainda em relação às condições de saúde, observou-se que os indivíduos com histórico de quedas apresentaram uma chance 3,24 vezes maior de apresentar autoavaliação negativa da saúde. Considera-se quedas uma síndrome geriátrica, multifatorial e heterogênea, apontada como a principal causa de morbidade e mortalidade entre os idosos ${ }^{28,35,36}$. Entre as consequências negativas das quedas está a inatividade física, em decorrência de dores e do medo de cair novamente, gerando declínio funcional que por sua vez gera insegurança, criando um ciclo autoperpetuante de imobilidade e incapacidade funcional, o qual tende a acentuar-se de acordo com a gravidade da ocorrência das quedas sofridas pelos idosos ${ }^{37}$.

A funcionalidade, por sua vez, também impacta a percepção de saúde dos idosos e fundamenta-se nos indicadores de mobilidade, equilíbrio e capacidade funcional, fatores intrinsecamente relacionados e que refletem potencialidades diferenciais para desempenhar as atividades de vida diária de maneira eficiente, como forma de garantir condições mínimas de sobrevivência e bem estar geral para os idosos ${ }^{28}$. Batistoni et al. ${ }^{32}$ relataram que o declínio funcional, uma das principais consequências de condições crônicas de saúde, representa um forte indicador da autoavaliação negativa da própria saúde. Neste estudo, verificou-se associação entre autoavaliação negativa da saúde com incapacidade para realização das atividades de vida diária, maior tempo para realização do teste Timed up \& go e autorrelato de menos energia para realizar as atividades de vida diária.

Uma limitação deste estudo é que a presença das doenças crônicas foi baseada no autorrelato dos participantes, o que pode ter comprometido a prevalência das mesmas.

Este estudo reforça a complexidade envolvida na percepção de saúde do idoso, pois a autoavaliação negativa da saúde foi determinada por fatores sociodemográficos, de saúde e funcionalidade. Neste estudo, os mais velhos, com recurso financeiro insuficiente, com histórico de quedas, hipertensão, doença neurológica, doença osteomioarticular, depressão, tempo de doença maior do que doze meses, pior funcionalidade, maior incapacidade nas atividades de vida diária e autorrelato de menos energia para realizar as atividades diárias tiveram maior chance de avaliar sua saúde como ruim ou péssima, ao passo que, os idosos que reportaram o uso de prótese dentária parcial ou total tiveram menor chance de avaliar negativamente sua saúde. Sendo muitos destes fatores potencialmente modificáveis, este estudo enfatiza a necessidade de um cuidado integral à saúde do idoso.

\section{REFERÊNCIAS}

1. Sander M, Oxlund B, Jespersen A, Krasnik A, Mortensen EL, Westendorp RGJ, et al. The challenges of human population ageing. Age Ageing. 2015;44(2):185-7

http://dx.doi.org/10.1093/ageing/afu189

2. Pinto RBR, Bastos LC. Abordagem das pesquisas em epidemiologia aplicada à gerontologia no Brasil: revisão da literatura em periódicos, entre 1995 e 2005. Rev Bras Epidemiol. 2007;10(3):361-9. http://dx.doi.org/10.1590/S1415-790X2007000300007

3. Geue C, Briggs A, Lewsey J, Lorgelly P. Population ageing and healthcare expenditure projections: new evidence from a time to death approach. Eur J Health Econ. 2014;15(8):885-96. http://dx.doi.org/10.1007/s10198-013-0543-7

4. Beard JR, Bloom DE. Towards a comprehensive public health response to population ageing. Lancet. 2015;385(9968):658-61. http://dx.doi.org/10.1016/S0140-6736(14)61461-6

5. Pimenta FA, Amaral CS, Torres HG, Rezende N. Autopercepção do estado de saúde em reformados e sua associação com o uso de serviços de saúde. Acta Med Port. 2010;23(1):101-6.

6. Pagotto V, Bachion MM, Silveira EA. Autoavaliação da saúde por idosos brasileiros: revisão sistemática da literatura. Rev Panam Salud Publica. 2013;33(4):302-10.

http://dx.doi.org/10.1590/S1020-49892013000400010

7. Bruin A, Picavet HSJ, Nossikov A. Health interview surveys: toward international harmonization of methods and instruments. Copenhagen: WHO; 1996

8. DeSalvo KB, Bloser N, Reynolds K, He J, Muntner P. Mortality prediction with a single general self-rated health question. A metaanalysis. J Gen Intern Med. 2006; 21(3):267-75. http://dx.doi.org/10.1111/j.1525-1497.2005.00291.x
9. Peres MA, Masiero AV, Longo GZ, Rocha GC, Matos IB, Najnie K, et al. Auto-avaliação da saúde em adultos no Sul do Brasil. Rev Saúde Pública. 2010;44(5):901-11.

http://dx.doi.org/10.1590/S0034-89102010000500016

10. Jóia LC, Ruiz T, Donalísio MR. Grau de satisfação com a saúde entre idosos do município de Botucatu, Estado de São Paulo, Brasil. Epidemiol Serv Saúde. 2008;17(3):187-9.

http://dx.doi.org/10.5123/S1679-49742008000300004

11. Freitas DHM, Campos FCA, Linhares LQ, Santos CR, Ferreira CB, Diniz BS, et al. Autopercepção de saúde e desempenho cognitivo em idosos residentes na comunidade. Rev Psiquiatr Clín. 2010;37(1):32-5.

http://dx.doi.org/10.1590/S0101-60832010000100007

12. Minas Gerais. Mais Noticias. Minas Gerais é o único do Sudeste com municípios com alta vulnerabilidade social. Disponível em: https://www.em.com.br/app/noticia/gerais/2015/09/01/interna_ gerais,684109/minas-gerais-e-o-unico-do-sudeste-com-municipioscom-alta-vulnerabilid.shtml. Acesso em: 01 nov. 2017

13. Veras RP, Souza CAM, Cardoso RF, Milioli R, Silva SD. Pesquisando populações idosas: a importância do instrumento e o treinamento de equipe: uma contribuição metodológica. Rev Saúde Pública. 1988;22(6):513-8.

http://dx.doi.org/10.1590/S0034-89101988000600008

14. Podsiadlo D, Richardson S. The timed "up \& go": a test of basic functional mobility for frail elderly persons. J Am Geriatr Soc. 1991;39(2):142-8.

http://dx.doi.org/10.1111/j.1532-5415.1991.tb01616.x

15. Fleming KC, Evans JM, Weber DC, Chutka DS. Pratical functional assessment of elderly persons: a primary-approach. Mayo Clin Proc. 1995;70(9):890-910. http://dx.doi.org/10.1016/S0025-6196(11)63949-9 
16. Bertolucci PHF, Brucki SMD, Campacci SR, Juliano Y. O MiniExame do Estado Mental em uma população geral: impacto da escolaridade. Arq Neuro-Psiquiatr. 1994;52(1):1-7. http://dx.doi.org/10.1590/S0004-282X1994000100001

17. Lima LCV, Bueno CMLB. Envelhecimento e gênero: a vulnerabilidade de idosas no Brasil. Rev Saúde Pesq. 2009;2(2):273-80.

18. Serbim AK, Figueiredo AEPL. Qualidade de vida de idosos em um grupo de convivência. Scientia Med. 2011;21(4):166-72.

19. Geib LTC. Determinantes sociais da saúde do idoso. Ciênc Saúde Coletiva. 2012; 17(1):123-33. http://dx.doi.org/10.1590/S1413-81232012000100015

20. Almeida VA, Mafra SCT, Silva EP, Kanso S. A Feminização da velhice: em foco as características socioeconômicas, pessoais e familiares das idosas e o risco social. Textos Contextos. 2015;14(10:115-31

http://dx.doi.org/10.15448/1677-9509.2015.1.19830

21. Soares MRP, Istoe RSC. Alfabetização e inclusão de pessoas idosas: uma proposta interdisciplinar mediada pelas tecnologias da informação e da comunicação. Rev Cient Interd. 2015;2(3):165-75. http://dx.doi.org/10.17115/2358-8411/v2n3a17

22. Instituto Brasileiro de Geografia e Estatística (IBGE). Pesquisa nacional por amostra de domicílios. síntese de indicadores sociais, 2015. Disponível em: http://ndonline.com.br/uploads/ global/materias/2015/12/04-12-2015-02-58-43-pesquisa-ibge. pdf. Acesso em: 18 jul. 2016.

23. Neri AL, Yassuda MS, Araújo LF, Eulálio MC, Cabral BE, Siqueira MEC, et al. Metodologia e perfil sociodemográfico, cognitivo e de fragilidade de idosos comunitários de sete cidades brasileiras: Estudo FIBRA. Cad Saúde Pública. 2013; 29(4):778-92 http://dx.doi.org/10.1590/S0102-311X2013000400015

24. Alves LC, Rodrigues RN. Determinantes da autopercepção de saúde entre idosos do Município de São Paulo, Brasil. Rev Panam Saúde Pública. 2005;17(5-6):333-41. http://dx.doi.org/10.1590/S1020-49892005000500005

25. Wickrama K, Mancini JA, Kwag K, Kwon J. Heterogeneity in multidimensional health trajectories of late old years and socioeconomic stratification: a latent trajectory class analysis. J Gerontol B Psychol Sci Soc Sci. 2013;68(2):290-7. http://dx.doi.org/10.1093/geronb/gbs111

26. Silva RJS, Smith-Menezes A, Tribess S, Rómo-Perez V, Virtuoso Júnior JS. Prevalência e fatores associados à percepção negativa da saúde em pessoas idosas no Brasil. Rev Bras Epidemiol. 2012:15(1):49-62. http://dx.doi.org/10.1590/S1415-790X2012000100005
27. Nunes APN, Barreto SM, Gonçalves LG. Relações sociais e autopercepção da saúde: Projeto Envelhecimento e Saúde. Rev Bras Epidemiol. 2012:15(2):415-28. http://dx.doi.org/10.1590/S1415-790X2012000200019

28. Borim FSA, Neri AL, Francisco PMSB, Barros MBA. Dimensões da autoavaliação de saúde em idosos. Rev Saúde Pública. 2014;48(5):714-22 http://dx.doi.org/10.1590/S0034-8910.2014048005243

29. Belém PLO, Melo RLP, Pedraza DF, Menezes TN. Autoavaliação do estado de saúde e fatores associados em idosos cadastrados na Estratégia Saúde da Família de Campina Grande, Paraíba. Rev Bras Geriatr Gerontol. 2016;19(2):265-76. http://dx.doi.org/10.1590/1809-98232016019.140206

30. Pagotto V, Nakatani AYK, Silveira EA. Fatores associados à autoavaliação de saúde ruim em idosos usuários do Sistema Único de Saúde. Cad Saúde Pública. 2011:27(8):1593-602. http://dx.doi.org/10.1590/S0102-311X2011000800014

31. Borim FSA, Barros MBA, Neri AL. Autoavaliação da saúde em idosos: pesquisa de base populacional no município de Campinas, São Paulo, Brasil. Cad Saúde Pública 2012:28(4):769-80. http://dx.doi.org/10.1590/S0102-311X2012000400016

32. Batistoni SST, Prestes SM, Cachioni M, Falcão DVS, Lopes A Yassuda MS, et al. Categorização e identificação etária em uma amostra de idosos brasileiros residentes na comunidade. Psicol Reflex Crít. 2015;28(3):511-21.

33. Alves LC. Determinantes da autopercepção de saúde dos idosos do município de São Paulo, 1999/2000. Dissertação (Mestrado) Universidade Federal de Minas Gerais. Belo Horizonte: 2004.

34. Keyes CL, Westerhof GJ. Chronological and subjective age differences in flourishing mental health and major depressive episode. Aging Ment Health. 2012;16(1):67-74. http://dx.doi.org/10.1080/13607863.2011.596811

35. Martinez DJ, KasI SV, Gill TM, Barry LC. Longitudinal association between self-rated health and timed gait among older persons. $J$ Gerontol B Psychol Sci Soc Sci. 2010;65(6):715-9. http://dx.doi.org/10.1093/geronb/gbp115

36. Pinto LLT, Leal Neto JS, Rocha SV, Vasconcelos LRC, Santos MC, Silva DAS. Indicadores de saúde entre idosos ativos e insuficientemente ativos residentes em áreas rurais. Rev Med (Ribeirão Preto) 2015:48(6):580-8 http://dx.doi.org/10.11606/issn.2176-7262.v48i6p580-588

37. Pinho TAM, Silva AO, Tura LFR, Silva MA, Gurgel SN, Smith AZF et al. Avaliação do risco de quedas em idosos atendidos em Unidade Básica de Saúde. Rev Esc Enferm USP. 2012:46(2):320-7. http://dx.doi.org/10.1590/S0080-62342012000200008 